\title{
POLYHEDRAL RESOLUTIONS OF ALGEBRAIC VARIETIES
}

\author{
BY
}

JAMES A. CARLSON ${ }^{1}$

\begin{abstract}
We give a method for constructing relatively small smooth simplicial resolutions of singular projective algebraic varieties. For varieties of dimension $n$, at most $n$ applications of the basic process yields a resolution of combinatorial dimension at most $n$. The object so obtained may be used to compute the mixed Hodge stucture of the underlying variety.
\end{abstract}

1. Introduction. To compute the mixed Hodge structure of a complex projective variety $Y$ one uses, following Deligne $[\mathbf{D}]$, a smooth simplicial resolution $[\varepsilon: X . \rightarrow Y]$. As one measure of the size of a resolution, one may take the combinatorial dimension:

$$
\operatorname{cdim} X .=\left\{\max p: X_{p} \neq 0\right\} .
$$

The purpose of this note is to give a method of constructing smooth simplicial resolutions which are small and sometimes rather efficient.

THEOREM 1.1. Let $Y$ be a complex projective variety of dimension $n$. Then $Y$ has a simplicial resolution of combinatorial dimension at most $n$.

The method described permits one to approach, from a single point of view, a number of $a d$ hoc constructions which have arisen in various applications. Three of these resolutions are described in the last section.

The objects by means of which the small resolutions are constructed are polyhedral spaces, which we describe informally now and formally in the next section. ${ }^{2}$ In rough terms such a space consists of a polyhedron $\mathscr{P}$, a topological space $X_{\sigma}$ for each face $\sigma$ of $\mathscr{P}$, and a continuous map $f_{\tau \sigma}: X_{\sigma} \rightarrow X_{\tau}$ for each inclusion of faces $(\tau<\sigma)$. The face maps must satisfy the cocycle condition

$$
f_{\nu \lambda}=f_{\nu \mu} \circ f_{\mu \lambda}
$$

whenever $\tau>\nu>\lambda$. As an example, consider the abstract mapping cyclinder, which we may give either as a diagram

$$
C(f, g)=[L \stackrel{f}{\leftarrow} M \stackrel{g}{\rightarrow} R],
$$

or as labelled version of the polyhedron $I=[0,1]$,

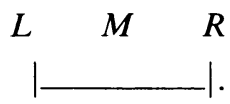

We say that $C(f, g)=C \mid I$ is a polyhedral space "over" the unit interval.

\footnotetext{
Received January 2, 1985.

1980 Mathematics Subject Classification. Primary 14C30.

${ }^{1}$ Partially supported by NSF Grant MCS 8102745 .

${ }^{2}$ The definition is due to Deligne.
} 
To every polyhedral space $X=X \mid \mathscr{P}$ is functorially associated an ordinary topological space, the realization. To define it, let $\phi_{\sigma \tau}: \tau \rightarrow \sigma$ be the linear inclusion of $\tau$ as a face of $\sigma$. Then the realization is the identification space $|X|=\left(X_{\sigma} \times \sigma\right) / \sim$ where the equivalence relation is given by

$$
\left(f_{\mu \lambda}\left(x_{\lambda}\right), t_{\mu}\right) \sim\left(x_{\lambda}, \phi_{\lambda \mu}\left(t_{\mu}\right)\right)
$$

wherever $\mu$ is a face of $\lambda$. The usual mapping cyclinder, mapping cone, and suspension are particular cases of the realization $|C(f, g)|$.

An augmentation $\varepsilon$ of a polyhedral space $X \mid \mathscr{P}$ by an ordinary topological space $Y$ is a system of maps $\varepsilon_{\sigma}: X_{\sigma} \rightarrow Y$ such that $\varepsilon_{\sigma}=\varepsilon_{\tau} \circ f_{\tau \sigma}$. Whenever $X \mid \mathscr{P}$ is augmented by $Y$ there is a canonical and functorial map $|\varepsilon|:|X.| \rightarrow Y$. When this map is a homotopy equivalence, we call it a polyhedral resolution of $Y$. If $X$ is smooth in the sense that each $X_{\sigma}$ is smooth, then $\varepsilon$ is a smooth polyhedral resolution. As an example of such, consider a projective variety $Y$ with singular locus $\Sigma$, let $\pi: \tilde{Y} \rightarrow Y$ be a resolution of singularities, and let $\tilde{\Sigma}$ be the preimage of $\Sigma$ in $Y$. There results a polyhedral variety

$$
C(i, \pi)=[\tilde{Y} \leftarrow \tilde{\Sigma} \rightarrow \Sigma]
$$

which is augmented by $Y$.

EXAMPLE [C]. Let $Y$ be a curve. Then the augmentation map looks like Figure 1.1. The remaining map $p$ is a canonical map $|X| \mathscr{P}|\rightarrow| \mathscr{P} \mid$ of the realization of a polyhedral space to its polyhedron. Here $p^{-1}(0) \cong \tilde{Y}, p^{-1}(0,1) \cong \tilde{\Sigma} \times(0,1)$, and $p^{-1}(1) \cong \Sigma$. Note that the fibers of $|\varepsilon|$ are either points or cones over parts of $\tilde{\Sigma}$. Since $|\varepsilon|$ has contractible fibers, it is a smooth polyhedral resolution.

It is easy to see that $C(i, \pi)$ is always a resolution, although not always smooth as in the example. If $y$ is a smooth point of $Y$, then the corresponding fiber of $|\varepsilon|$ is the realization of

$$
\varepsilon^{-1}(y)=[\{y\} \leftarrow \varnothing \rightarrow \varnothing],
$$

which is a point. If $y$ is a singular point, then the fiber is the realization of

$$
\varepsilon^{-1}(y)=\left[\pi^{-1}(y) \leftarrow \pi^{-1}(y) \rightarrow\{y\}\right],
$$

which is the cone over $\pi^{-1}(y)$ with vertex $y$. Since the fiber of $|\varepsilon|$ is contractible in either case, $\varepsilon$ is a polyhedral resolution.
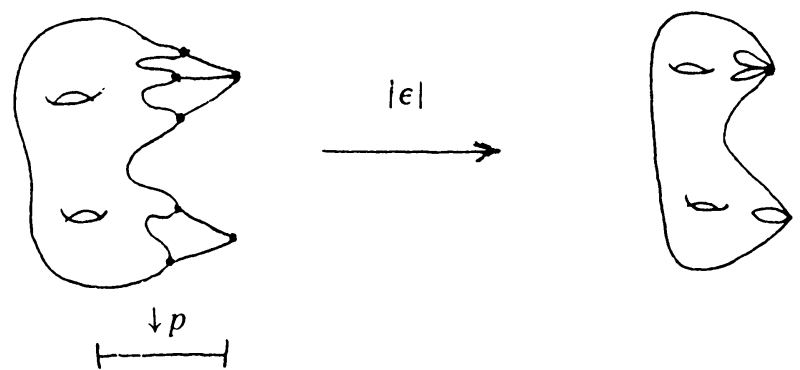

FIGURE 1.1 
The main idea of this paper is based on the observation that, although the singular locus of

$$
Y \mid I \underset{\operatorname{def}}{=} C(i, \pi)
$$

may not be empty, it must be of dimension smaller than that of the singular locus of $Y$. To produce smooth resolutions, one would, therefore, like to repeat the construction just given with $Y \mid I$ instead of $Y$ as starting point. For this it suffices to be able to

(i) desingularize polyhedral spaces,

(ii) form the mapping cylinder in the category of polyhedral spaces, with a certain control on the size of the singular loci produced.

Thus, step (i) removes singularities at the cost of changing the topology of the realization, while step (ii) restores the correct topology at the cost of introducing lower-dimensional singularities.

To summarize, a tower of resolutions

$$
Y \leftarrow Y|I \leftarrow Y| I^{2} \leftarrow \cdots \leftarrow Y \mid I^{m}
$$

over the unit $r$-cubes $I^{r}$ will result, where the dimension of the singular locus of $Y \mid I^{r}$ is at most $n-r$ and where the right-most space is smooth. Since the process introduces certain redundancies, we also give a method ("consolidation", see §2) which will be used to constuct the examples of the last section.

$\S \S 2$ and 3 contain foundational and collateral material, while $\S \S 4,5$, and 6 contain the essentials.

Similar results have been obtained by Guillen, Navarro Aznar, and Puerta of Barcelona in their work Cubical hyper-resolutions.

2. Polyhedral spaces. We shall now discuss polyhedral spaces in some detail. The theory closely parallels that of simplicial spaces [D]. A polyhedron $\sigma$ is by definition the convex hull of a finite point set in a real vector space. A face $\tau$ of $\sigma$ is the intersection of $\sigma$ with a supporting hyperplane. To denote that $\tau$ is a face of $\sigma$, we write $\tau<\sigma$, with $\tau \leqslant \sigma$ admitting the additional possibility that $\tau=\sigma$. A polyhedral complex $\mathscr{P}$ is a collection of polyhedra in a vector space $V$ such that

(i) if $\sigma \in \mathscr{P}$ and $\tau \leqslant \sigma$, then $\tau \in \mathscr{P}$,

(ii) if $\sigma, \tau \in \mathscr{P}$, then $\sigma \cap \tau \leqslant \sigma$.

The span of such a complex is the subset of $V$ defined by $|\mathscr{P}|=\bigcup_{\sigma \in \mathscr{P}} \sigma$.

A polyhedral complex defines a category $\mathscr{C} \mathscr{P}$ whose objects are the elements $\sigma$ of $\mathscr{P}$ and whose morphisms are the face maps, i.e. the linear inclusions $\phi_{\sigma \tau}: \tau \rightarrow \sigma$ defined whenever $\tau<\sigma$. A morphism $f: \mathscr{C} \mathscr{P} \rightarrow \mathscr{C} \mathscr{Q}$ is a morphism in the ordinary sense subject to the restriction that $\operatorname{dim} f(\sigma) \leqslant \operatorname{dim}(\sigma)$. By virtue of this restriction, vertices must go to vertices. Morphisms do in fact come about geometrically, as the next lemma shows. When necessary to make the distinction, we shall write " $\sigma$ " when $\sigma$ is viewed as an object of $\mathscr{C} \mathscr{P}$, and " $|\sigma|$ " when it is viewed as a convex body.

LEMMA 2.1. Let $f: \mathscr{C P P} \rightarrow \mathscr{C} 2$ be a morphism of polyhedral complexes. Then there is a continuous map $F:|\mathscr{P}| \rightarrow|\mathscr{Q}|$ which induces $f$ in the sense that $F(|\sigma|)=|f(\sigma)|$. 
Proof. Let $\mathscr{P}_{n}=\{\sigma \in \mathscr{P} \mid \operatorname{dim} \sigma \leqslant n\}$ be the $n$-skeleton of $\mathscr{P}$. Since vertices go to vertices, $f: \mathscr{C}_{\mathscr{P}_{0}} \rightarrow \mathscr{C} \mathscr{Q}$ determines $F_{0}:\left|\mathscr{P}_{0}\right| \rightarrow|\mathscr{Q}|$. Assume that $F_{n}:\left|\mathscr{P}_{n}\right| \rightarrow|\mathscr{Q}|$ has been constructed with the required properties, and let $\sigma$ be an $(n+1)$-polyhedron of $\mathscr{P}$. Set $\tau=f(\sigma)$, and let $\hat{\sigma}, \hat{\tau}$ be the respective barycenters. Given $x$ in the boundary of $\sigma$, let $\gamma_{x}:[0,1] \rightarrow$ be the unique affine map with $\gamma_{x}(0)=\hat{\sigma}$ and $\gamma_{x}(1)=x$. For $y$ in the boundary of $\tau$ let $\bar{\gamma}_{y}$ be the analogous map. Define $F_{n+1}$ on $\sigma$ by $F_{n+1}\left[\gamma_{x}(t)\right]=$ $\bar{\gamma}_{1}(t)$ with $y=F_{n}(x)$.

A polyhedral object in a category $\mathscr{C}$ is a functor from $\mathscr{P}$ to $\mathscr{C}$. We say that $X$ is a polyhedral object in $\mathscr{C}$ over $\mathscr{P}$, and we write $X \mid \mathscr{P}$ when necessary to stress the identity of the domain category. A polyhedral object is given by objects $X_{\sigma}=X(\sigma)$ gotten by applying $X$ to the objects of $\mathscr{P}$ and related by morphisms $f_{\sigma \tau}: X_{\tau} \rightarrow X_{\sigma}$ gotten by applying $X$ to the morphisms of $\mathscr{P}$. The cocyle condition of section one is forced upon us by functoriality. A morphism of polyhedral objects $M: X|\mathscr{P} \rightarrow Y| \mathscr{Q}$ is given by

(i) an underlying morphism $m: \mathscr{C} \mathscr{P} \rightarrow \mathscr{C} \mathscr{Q}$,

(ii) morphisms $M_{\sigma}: X_{\sigma} \rightarrow Y_{m \sigma}$ sucht hat the diagrams below commute:

$\begin{array}{rll}X_{\sigma} & \stackrel{M_{\sigma}}{\rightarrow} & Y_{m \sigma} \\ f_{\tau \sigma}^{X} \downarrow & & \downarrow f_{m \tau, m \sigma}^{Y} \\ X_{\tau} & \vec{M}_{\tau} & Y_{m \tau}\end{array}$

An ordinary object $Y$ in $\mathscr{C}$ can be thought of as a polyhedral object with a one-point polyhedron, $Y \mid *$. With this convention, we define an augmentation of $X \mid \mathscr{P}$ by $Y$ as a morphism $\varepsilon: X|\mathscr{P} \rightarrow Y| *$. Often the "star" will be omitted.

A polyhedral space can now be defined as a contravariant polyhedral object in $\mathscr{C}$, the category of topological spaces. If the target category is smaller, say the category of algebraic varieties, we may speak of a polyhedral variety in the same fashion one speaks of polyhedral manifolds, etc.

There are several elementary but important functorial constructs for polyhedral spaces. The first of these is the reduction, defined by

$$
R(X \mid)=\left(\coprod_{\sigma \in P} X_{\sigma}\right) / \sim
$$

where the equivalence relation on the disjoint union is that given by

$$
x_{\sigma} \sim x_{\tau} \text { if } x_{\sigma}=f_{\sigma \tau}\left(x_{\tau}\right) .
$$

Note that $X \mid \mathscr{P}$ is canonically augmented by $R(X \mid \mathscr{P})$.

Example 2.1. Let $C=[X \leftarrow A \rightarrow *]$ then $R(C)$ is $X$ with $A$ collapsed to a point.

ExAmple 2.2. Let $\mathscr{U}=\left\{U_{i} \mid i \in A\right\}$ be a cover of $Y$. For each subset $S \subseteq A$, set $U_{S}=\cup_{i \in S} V_{i}$. Let $V$ be a vector space with $A$ as basis, let $|S|$ be the convex hull of $S$, and let $\mathscr{P}=\left\{|S|: U_{S} \neq \varnothing\right\}$ be the polyhedral complex defined by the cover. The nerve of $U$ is the polyhedral space over $\mathscr{P}$ defined by the correspondence $N \mathscr{U}$ : $S \rightarrow U_{S}$. The nerve is augmented by $Y$, and its reduction is homeomorphic to $Y$ :

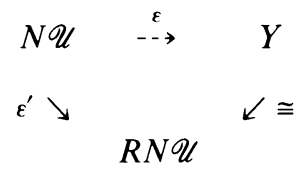


One can also define copolyhedral spaces as covariant functors from $\mathscr{C} \mathscr{P}$ to top. Recalling the scholastic distinction between $\sigma$ and $|\sigma|$, the "identity" functor $I$ : $\sigma \rightarrow|\sigma|$ defines such an object. Although we should properly write $I\left(\phi_{\sigma \tau}\right)=\left|\phi_{\sigma \tau}\right|$, this convention will usually be broken. The reduction functor makes sense in the covariant world as well, so that one has a canonical homomorphism $R(I \mid \mathscr{P}) \rightarrow|\mathscr{P}|$ which is given by the augmentation. As a slightly fancier example we define a model for $\mathscr{P}$ as a copolyhedral space $M \mid \mathscr{P}$ endowed with a homeomorphism $\Phi: M|\mathscr{P} \rightarrow I| \mathscr{P}$ so that $R(M \mid \mathscr{P})$ is a topological space endowed with a canonical homeomorphism $R(M \mid \mathscr{P}) \rightarrow|\mathscr{P}|$. Following are two examples, the second of which will be used later.

(a)
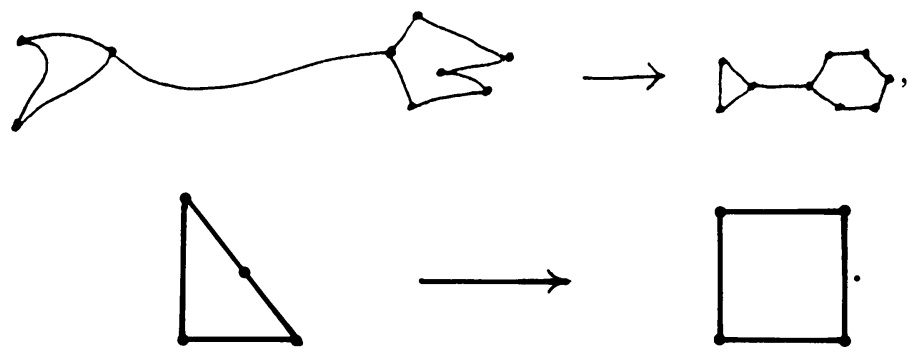

A realization of $X \mid \mathscr{P}$ relative to a model $M \mid \mathscr{P}$ is defined as

$$
\left.|X| \mathscr{P}\right|_{M}=\left(\coprod X_{\sigma} \times M_{\sigma}\right) / \sim
$$

where the equivalence is given by $\left(x_{\lambda}, m_{\lambda \mu} t_{\mu}\right) \sim\left(f_{\mu \lambda} x_{\lambda}, t_{\mu}\right)$. Given a morphism $G=\left(G^{\prime}, G^{\prime \prime}\right)$ of $(X|\mathscr{P}, M| \mathscr{P})$ to $(Y|\mathscr{Q}, N| \mathscr{Q})$, there is a functorially defined morphism of realizations $|G|:|X|_{M} \rightarrow|Y|_{N}$ induced by $\left(x_{\sigma}, t_{\sigma}\right) \rightarrow\left(G_{\sigma}^{\prime} x_{\sigma}, G_{\sigma}^{\prime \prime} t_{\sigma}\right)$ on the level of the disjoint unions. In what follows we shall generally omit the subscript which identifies the model used, particularly when it is the identity model. This omission is partially justified by the fact that different models give homeomorphic realizations.

A polyhedral space is always augmented by its realization, although not canonically so. To define one augmentation, let $\hat{\sigma}$ denote the barycenter of $\sigma$, and map $X_{\sigma}$ to $X_{\sigma} \times\{\hat{\sigma}\}$, where the latter is viewed in $R(X \mid \mathscr{P})$.

A polyhedral resolution is a morphism

$$
\varepsilon: X|\mathscr{P} \rightarrow Y| *
$$

which induces a homotopy equivalence on the level of realizations. As a trivial but alliterative example, one notes that $X \mid \mathscr{P}$ resolves its realization. As a somewhat less trivial example, we note that the nerve of a cover is a polyhedral resolution of the space covered. To see that this is indeed the case, define the carrier of a point $p$ in $Y$ to be the simplex $C(p)=\left\{i \in A \mid p \in U_{i}\right\}$. Thus, $C(p) \in \mathscr{P}$, and $|C(p)| \subseteq|\mathscr{P}|$. The important point is now that the fiber $|\varepsilon|^{-1}(p)$ is the simplex $|C(p)|$, hence contractible, so that $|\varepsilon|$ is a homotopy equivalence, as required.

The realization and the reduction are related by the commutative diagram below:

$$
\begin{array}{ccc} 
& X \mid \mathscr{P} & \\
\varepsilon \swarrow & & \searrow \varepsilon^{\prime} \\
|X| \mathscr{P} \mid & \rightarrow & R(X \mid \mathscr{P})
\end{array}
$$


Although the two constructs are homotopy equivalent for nerves of covers, they are not homotopy equivalent in general, as the following example shows:

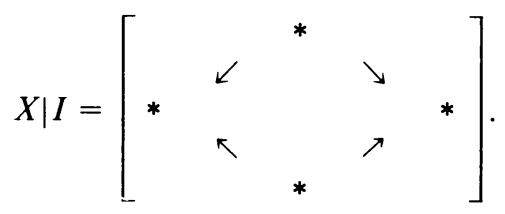

The realization is a circle, while the reduction is a point.

We end this section with some remarks on subdivisions of polyhedral complexes which will prove useful at the very end. Let $\mathscr{P}$ and $\mathscr{P}^{\prime}$ be complexes with the same span, and define, for each $\sigma$ in $\mathscr{P}$, the set

$$
\mathscr{P}_{\sigma}^{\prime}=\left\{\tau \in \mathscr{P}^{\prime} \mid \tau \leqslant \sigma\right\} .
$$

If $\mathscr{P}^{\prime}$ is a subcomplex such that $\left|\mathscr{P}_{\sigma}^{\prime}\right|=|\sigma|$ for each $\sigma$, then we say that is a subdivision of $\mathscr{P}$. In this case there is a smallest polyhedron $C(\tau) \in \mathscr{P}$, the carrier, which contains $\tau \in \mathscr{P}^{\prime}$. The subdivision of a polyhedral space $X \mid \mathscr{P}$ by $\mathscr{P}^{\prime}$ is then the polyhedral space $X^{\prime} \mid \mathscr{P}^{\prime}$ defined by the relations

$$
X_{\tau}^{\prime}=X_{C(\tau)}, \quad F_{\lambda \nu}^{X^{\prime}}=F_{C(\lambda) C(\nu)}^{X} .
$$

If $\varepsilon: X|\mathscr{P} \rightarrow Y| *$ is an augmentation, then there is an induced augmentation $\varepsilon^{\prime}: X^{\prime}\left|\mathscr{P}^{\prime} \rightarrow Y\right| *$ given by

$$
\left[\varepsilon^{\prime}: X_{\tau}^{\prime} \rightarrow Y\right]=\left[\varepsilon_{C(\tau)}: X_{C(\tau)} \rightarrow Y\right]
$$

Moreover, there is a canonical homeomorphism of realizations which is compatible with the augmentation:

$$
\begin{array}{cccc}
|X| \mathscr{P} \mid & \stackrel{\cong}{\rightarrow} & |X| \mathscr{P} \mid \\
\left|\varepsilon^{\prime}\right| \searrow & & \swarrow|\varepsilon| \\
& Y &
\end{array}
$$

The subdivision of polyhedral spaces using the labelled polyhedron representation is illustrated in Figure 2.2. The polyhedral space $Y \mid S$ over the square is obtained by subdividing the space $X \mid T$ over the triangle. Conversely, we say that $X \mid T$ arises from $Y \mid S$ by unsubdivision or consolidation. Consolidation will be used to simplify

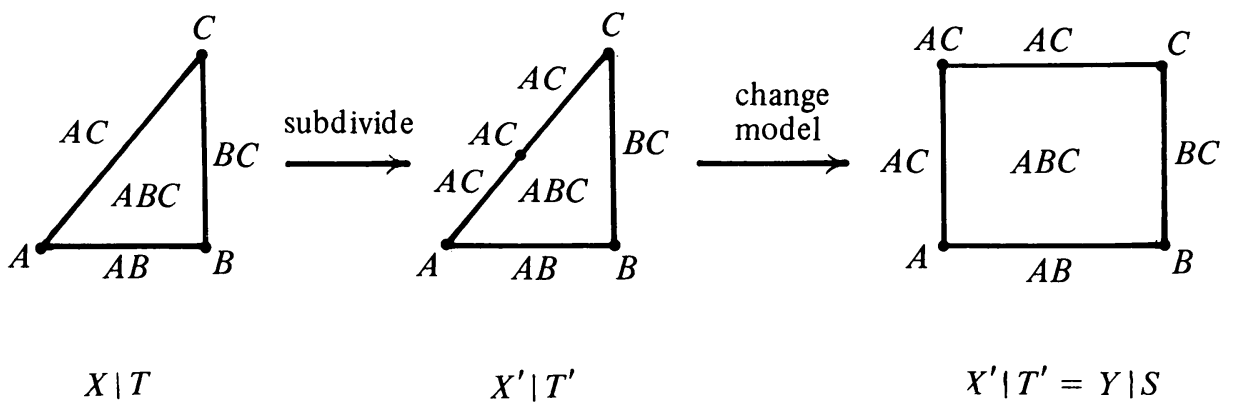

Figure 2.2 
polyhedral resolutions. For the moment, we note that every polyhedral space admits a simplicial subdivision, i.e. one such that each polyhedron $\tau$ is an unoriented simplex.

3. Simplicial polyhedral spaces and their cohomology. In this section we observe that an orientation of a simplicial polyhedral complex defines a simplicial space $X \mid \Delta_{N}$ from a polyhedral space $X \mid \mathscr{P}$. We then recall the cohomology theory of such objects, following Deligne [D].

An orientation of a simplicial polyhedral complex is a bijection $\beta: \mathscr{P}_{0} \rightarrow\{0, \ldots, N\}$ defined on the set of vertices. An orientation associates an increasing sequence to each $p$-simplex, $I=\left(i_{0}, \ldots, i_{p}\right)=\beta(\sigma)$, where $\left\{\beta^{-1}\left(i_{0}\right), \ldots, \beta^{-1}\left(i_{p}\right)\right\}$ is the set of vertices of $\sigma$. For each increasing sequence $I$ define $\delta_{j}(I)=\left(i_{0}, \ldots, \hat{i}_{j}, \ldots, i_{p}\right)$, where the superscript denotes omisson. Set

$$
X_{I}=X_{\beta^{-1}(I)}, \quad X[p]=\coprod_{|I|=p} X_{I},
$$

where $\left|\left(i_{0}, \ldots, i_{p}\right)\right|=p$. Define maps

$$
\delta_{j}: X[p] \rightarrow X[p-1] \quad(j=0, \ldots, p)
$$

by

$$
\left[\delta_{j}: X_{I} \rightarrow X_{\delta_{j}(I)}\right]=\coprod f_{\delta_{j}(I) I} .
$$

There results an assembly of maps and spaces

$$
X \mid \Delta_{N}=\left[X[0] \underset{\delta_{1}}{\delta_{0}} X[1] \leftleftarrows \cdots\right]
$$

To place such an object in its proper context $[\mathbf{D}]$, let $[p]=\{0,1, \ldots, p\}$, and let $\Delta_{N}$ be in the category whose objects are $[0], \ldots,[N]$, and whose morphisms are generated by the order-preserving injections $\delta_{j}:[p] \rightarrow[p+1]$ defined by $j \notin \delta_{j}[p]$. A semisimplicial object in a category $\mathscr{C}$ of combinatorial dimension $N$ is then a functor $X: \Delta_{N} \rightarrow \mathscr{C}$, usually written $X \mid \Delta_{N}$. We shall generally omit the prefix "semi". The construction given in the preceding paragraph associates a simplicial space $X \mid \Delta_{N}$ to an oriented simplicial polyhedral space $X \mid \mathscr{P}$.

The virtue of simplicial spaces is that they admit a natural cohomology theory relative to functors $K: \mathscr{S T} \rightarrow K A$ from a subcategory of topological spaces to a category of complexes in an abelian category $A$. Following Deligne [D], one defines a cosimplicial complex

$$
K X \mid \Delta_{N}=\left[K\left(X_{0}\right) \underset{\delta^{1}}{\stackrel{\delta^{0}}{\rightrightarrows}} K\left(X_{1}\right) \rightrightarrows\right]
$$

and from it a single complex

$$
s K\left(X \mid \Delta_{N}\right)=\bigoplus_{p} K(X[p])[p],
$$


where one uses the convention $(L[p])^{q}=L^{p+q}$ for shifting degrees of a complex. The differential of $(s K)^{p}$ is given by $D_{p}(-1)^{p} d_{p}+\delta_{p}^{*}$ where $d_{p}$ is the differential of $K(X[p])$ and where $\delta_{p}^{*}=\sum_{i=0}^{p}(-1)^{i} \delta^{i}$ is the simplicial differential. The cohomology of $X \mid \Delta_{N}$ relative to $K$ is then defined by

$$
H\left(X \mid \Delta_{N}, K\right)=H\left(s K\left(X \mid \Delta_{N}\right)\right) \text {. }
$$

The simplicial filtration

$$
W^{\prime} s K=\bigoplus_{p \geqslant l} K(X[p])[p]
$$

defines a spectral sequence emanating from ordinary cohomology and abutting to the simplicial cohomology:

$$
E_{2}^{p q}=H^{q}(X[p], K)=H^{p+q}\left(X \mid \Delta_{N}, K\right) .
$$

We now define the cohomology of $X \mid \mathscr{P}$ to be that of the simplicial space $X \mid \Delta_{N}$ deduced from but independent of an orientation of $\mathscr{P}$ :

$$
H(X \mid \mathscr{P}, K):=H\left(X \mid \Delta_{N}, K\right) .
$$

If $F: X|\mathscr{P} \rightarrow Y| \mathscr{P}$ is a morphism which is functorial for $K$, then there is an induced map

$$
F^{*}: H(Y \mid \mathscr{P}, K) \rightarrow H(X \mid \mathscr{P}, K) .
$$

In particular, if we choose $K$ to be the functor of singular cochains, then we obtain a singular cohomology theory for polyhedral spaces which is functorial for continuous morphisms. The following result shows that the definitions made are reasonable:

THEOREM 3.1. $\kappa: X|\mathscr{P} \rightarrow| X|\mathscr{P}|$ be the canonical augmentation. Then the induced map of singular cohomology

$$
\kappa^{*}: H(|X| \mathscr{P} \mid) \rightarrow H(X \mid \mathscr{P})
$$

is an isomorphism.

Proof of THE THEOREM. The argument is based on the existence of a special cover for $|X| \mathscr{P} \mid$. Define first a polyhedral space $\mathscr{P} \mid \mathscr{P}$ by assigning to each object $\sigma$ of $\mathscr{P}$ a one-point topological space $\{\sigma\}$, and observe that there is a canonical homeomorphism $|\mathscr{P}| \mathscr{P}|\rightarrow| \mathscr{P} \mid$. The morphism $X|\mathscr{P} \rightarrow \mathscr{P}| \mathscr{P}$ defined by $X_{\sigma} \rightarrow\{\sigma\}$ therefore induces a canonical map $p:|X| \mathscr{P}|\rightarrow| \mathscr{P} \mid$. (See Figure 1.1.) Choose an orientation of $\mathscr{P}$ and let $t$ be the associated barycentric coordinate vector for points of $\sigma$. Define a metric on $\sigma$ by

$$
d_{\sigma}(x, y)=\max \left|t_{\sigma}^{i}(x)-t_{\sigma}^{i}(y)\right|,
$$

and let $d(x, y)$ be a metric on $|\mathscr{P}|$ which agrees with $d_{\sigma}(x, y)$ on $\sigma$. For small $\delta$ let

$$
U_{i}=\{x \in|\mathscr{P}|: d(x, i)<1-\delta\},
$$

where $i$ is a vertex of $\mathscr{P}$. The nerve of this canonical cover of $|\mathscr{P}|$ has $\mathscr{P}$ as polyhedron. Indeed, the open set $U_{I}=U_{i_{0}} \cap \cdots \cap U_{i_{r}}$ is a contractible open neighborhood of $\hat{I}$, the barycenter of the oriented simplex $I$. The required open cover of $|X| \mathscr{P} \mid$ is then the pullback of the canonical cover of $|\mathscr{P}|$, given by the sets 
$\tilde{U}_{i}=p^{-1}\left(U_{i}\right)$. Note that there is an inclusion

$$
j: X|\mathscr{P} \rightarrow N \tilde{\mathscr{U}}| \mathscr{P}
$$

for the nerve defined by sending $X_{I}$ to $p^{-1}(\hat{I})$, and there is a retraction

$$
r: N \tilde{\mathscr{U}}|\mathscr{P} \rightarrow X| \mathscr{P}
$$

defined by a suitable projection of $\tilde{U}_{I}=p^{-1}\left(U_{I}\right)$ onto $X_{I}$ identified with $p^{-1}(\hat{I})$. Since both $i$ and $r$ are homotopy equivalences of polyhedral spaces, the cohomology groups of $X \mid \mathscr{P}$ and $N \tilde{\mathscr{U}} \mid \mathscr{P}$ are canonically isomorphic.

Because the nerve of a cover is augmented by the space covered, one has the following commutative diagram:

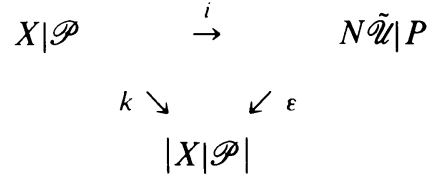

Since $k^{*}=i^{*} \varepsilon^{*}$ on cohomology with $i^{*}$ an isomorphism by the previous paragraph, it suffices to know that $\varepsilon^{*}$ is an isomorphism-an immediate consequence of the Mayer-Vietoris spectral sequence. For a cover with only two open sets as in Figure 1.1 , the result follows from the usual Mayer-Vietoris sequence. The nerve in this case is given by the three open sets $\tilde{U}_{0}=p^{-1}[0,2 / 3), \tilde{U}_{1}=p^{-1}(1 / 3,1]$, and $\tilde{U}_{01}=p^{-1}(1 / 3,2 / 3)$. To conclude, the theorem is true because a polyhedral resolution $X \mid \mathscr{P}$ of $Y$ is homotopy equivalent to the nerve of an open cover $|X| \mathscr{P} \mid$, a space which is in turn homotopy equivalent to $Y$. Thus, a polyhedral resolution of $Y$ is, up to homotopy, the nerve of an ordinary open cover of $Y$; it is a "generalized nerve".

4. Resolution of singularities. Our next task is to investigate the extent to which polyhedral varieties admit resolutions of singularities. The first result is general but weak:

THEOREM 4.1. For every polyhedral variety $Y \mid \mathscr{P}$ there exists an epimorphism $\pi: X|\mathscr{P} \rightarrow Y| \mathscr{P}_{\text {with }} X$ smooth and with $\operatorname{dim} Y_{\sigma}=\operatorname{dim} Y_{\sigma}$ for all $\sigma$.

To do better, we restrict the class of objects:

DEFINITION 4.2. A polyhedral space $Y \mid \mathscr{P}$ is epimorphic if all face maps are epimorphisms.

THEOREM 4.3. For every epimorphic polyhedral variety $Y \mid \mathscr{P}$ there exists a birational morphism $\pi: X|\mathscr{P} \rightarrow Y| \mathscr{P}$ with $X$ smooth and epimorphic.

A birational morphism $\pi$ is, of course, one such that each $\pi_{\sigma}$ is birational. We shall give the proofs after discussing in some detail the notions of the center and the exceptional locus of a morphism in the polyhedral category.

For ordinary varieties, the center of an epimorphism $\phi: X \rightarrow Y$ is the subspace $B$ of $Y$ above which $\phi$ is not an isomorphism, and the exceptional locus is the pullback to $X: E=\phi^{-1}(B)$. Since the correspondence $\sigma \rightarrow B\left(\phi_{\sigma}\right)$ does not in general define a polyhedral object, we must define the center of $\phi$ as the smallest polyhedral subspace containing the $B\left(\phi_{\sigma}\right)$ :

$$
B_{\sigma}=\bigcup_{\sigma \leqslant \tau} f_{\sigma \tau}\left[B\left(\phi_{\tau}\right)\right]
$$


where $f_{\sigma \sigma}$ is, by definition, the identity map. The exceptional locus is then defined by $E \phi=\phi^{-1}(B)$. Because the inverse image of a polyhedral subspace under an epimorphism is again a polyhedral subspace, this makes sense.

One measure of the size of a polyhedral space is the geometric dimension:

$$
\operatorname{gdim}(A \mid \mathscr{P})=\max \left\{\operatorname{dim} A_{\sigma} \mid \sigma \in \mathscr{P}\right\} .
$$

With respect to this measure the center and exceptional locus display some restraint:

Proposition 4.4. Let $f: X|\mathscr{P} \rightarrow Y| \mathscr{P}$ be a birational morphism of polyhedral varieties, and let $n=\operatorname{gdim}(X \mid \mathscr{P})$. Then

(i) $\operatorname{gdim}(B)<n$,

(ii) if $Y$ is epimorphic, then $\operatorname{gdim}(E)<n$.

Proof. (i) It suffices to show that $\operatorname{dim} f_{\sigma \tau}\left(B_{\tau}\right)<n$ for all $\sigma$ and $\tau$ with $\sigma \leqslant \tau$. Since $\phi_{\tau}$ is birational, $B\left(\phi_{\tau}\right)$ is a proper algebraic subset of $Y_{\tau}$, so that

$$
\operatorname{dim} f_{\sigma \tau} B\left(\phi_{\tau}\right)<\operatorname{dim} Y_{\tau} \leqslant n .
$$

Thus, $\operatorname{dim} B_{\sigma}$ is also less than $n$.

(ii) It suffices to show that $\operatorname{dim} \phi_{\sigma}^{-1} f_{\sigma \tau} B\left(\phi_{\tau}\right)<n$. If $f_{\sigma \tau} B\left(\phi_{\tau}\right)$ is a proper subset of $Y_{\sigma}$, then $\phi_{\sigma}^{-1} f_{\sigma \tau} B\left(\phi_{\tau}\right)$ is a proper subset of $X_{\sigma}$, so then inequality holds. If, on the contrary, $\operatorname{dim} f_{\sigma \tau} B\left(\phi_{\tau}\right)=\operatorname{dim} Y_{\sigma}$, then $\operatorname{dim} Y_{\sigma}<\operatorname{dim} X_{\tau} \leqslant n$ because $Y$ is epimorphic. But then $\operatorname{dim} \phi_{\sigma}^{-1} f_{\sigma \tau} B\left(\phi_{\tau}\right) \leqslant \operatorname{dim} X_{\sigma}=\operatorname{dim} Y_{\sigma}<n$.

Because it will be necessary to resolve the singularities of the center and exceptional locus of a morphism, we need variant constructions of these loci which are epimorphic but which still satisfy the previous proposition. To this end, let $Y \mid \mathscr{P}$ be epimorphic, and define formal inverse face operators $f_{\lambda \mu}^{Y}=\left(f_{\mu \lambda}^{Y}\right)^{-1}$ where $\mu \leqslant \lambda$ and where these are viewed as operators on subsets. Because of the set-theoretic identity $g \circ g^{-1}(A)=A$ for surjections, the cocycle rule extends to give $f_{\lambda \mu}^{Y} f_{\mu \nu}^{Y}=\lambda_{\lambda \nu}^{Y}$ where $f_{\lambda \mu}$ is a face map and where $f_{\mu \nu}$ is either a face map or an inverse face map. In other works, $\lambda \leqslant \mu$ and $\mu \sim \nu$, where the last expression means $\mu \leqslant \nu$ or $\mu \geqslant \nu$. The variant center is now defined by

$$
\bar{B}_{\sigma}=\bigcup_{\sigma \sim \tau} f_{\sigma \tau} B\left(\phi_{\tau}\right)
$$

To see that this does indeed give an epimorphic subspace of $Y \mid \mathscr{P}$, it suffices to show that $f_{\sigma \tau} \bar{B}_{\tau}=\bar{B}_{\sigma}$ when $\sigma \leqslant \tau$ :

$$
\begin{aligned}
f_{\sigma \tau} \bar{B}_{\tau} & =f_{\sigma \tau}\left[\bigcup_{\tau \sim \lambda} f_{\tau \lambda} B\left(\phi_{\lambda}\right)\right] \\
& =\bigcup_{\tau \sim \lambda} f_{\sigma \tau} f_{\tau \lambda} B\left(\phi_{\lambda}\right)=\bigcup_{\sigma \sim \lambda} f_{\sigma \lambda} B\left(\phi_{\lambda}\right),
\end{aligned}
$$

where the last equality uses the extended cocycle relation. The variant exceptional locus is then defined by $\bar{E}=\phi^{-1} \bar{B}$. Because the inverse image of an epimorphic space under an epimorphism is itself epimorphic, so must be $\bar{E}$.

Proposition 4.5. Let $\pi: X \rightarrow Y$ be a birational map of epimorphic polyhedral spaces. Then $\operatorname{gdim} \bar{B}<n$ and $\operatorname{gdim} \bar{E}<n$, where $n=\operatorname{gdim} Y$ as before. 
Proof. The inequality for $\bar{B}$ is proved much as before. It suffices to observe that if $f_{\sigma \tau}$ is an inverse face map, then $f_{\sigma \tau}\left(B_{\tau}\right)$, as the preimage of a proper subspace under an epimorphism, is a proper subspace of $Y_{\sigma}$.

For $\bar{E}$, one proceeds as before, adjoining the observation that if $f_{\sigma \tau}^{Y}$ is an inverse face map, then

$$
\phi_{\sigma}^{-1}\left(f_{\sigma \tau}^{Y}\right)\left(B_{\tau}\right)=\phi_{\sigma}^{-1}\left(f_{\tau \sigma}^{Y}\right)^{-1}\left(B_{\tau}\right)=\left(f_{\tau \sigma}^{Y} \phi_{\sigma}\right)^{-1}\left(B_{\tau}\right)=\left(\phi_{\tau} f_{\tau \sigma}^{X}\right)^{-1}\left(B_{\tau}\right),
$$

as a result of which the set in question is the inverse image of a proper subspace under a surjection, hence is itself a proper subspace.

The proofs of the theorems asserted at the beginning of this section rely on an extension lemma for morphisms. To describe the lemma, let $P$ be a polyhedron, let $\mathscr{F} P$ be the complex consisting of $P$ and its faces, and let $\partial \mathscr{F} P$ be the boundary complex obtained by deleting $P$. When there is no danger of confusion, we write $P$ and $\partial P$ for $\mathscr{F} P$ and $\partial \mathscr{F} P$.

Lemma 4.6. Let $\phi: X|\partial P \rightarrow Y| P$ be a morphism. Then there is a canonical commutative diagram extending $X$ and $\phi$ to $P$

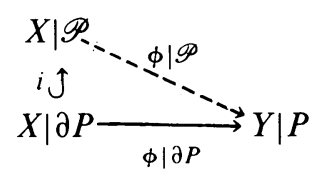

By this we mean in particular that $i_{\sigma}$ is the identity. In addition,

(i) if $\phi \mid \partial P$ is an epimorphism, then so is $\phi \mid P$,

(ii) if both $\phi \mid \partial P$ and $Y \mid P$ are epimorphic, then so is $X \mid P$.

Proof of Lemma 4.6. Let $F_{1}, \ldots, F_{n}$ be an ordering of the faces of $P$ of codimension one, and set

$$
X_{P}=\left\{\left(y_{P}, x_{1}, \ldots, x_{n}\right) \in Y_{P} \times \Pi X_{F_{i}} \mid \phi_{F_{i}}\left(x_{i}\right)=f_{F_{i} P}^{Y}\left(y_{P}\right)\right\} .
$$

The projections on the factors of the Cartesian product give maps

$$
\phi_{P}: X_{P} \rightarrow Y_{P}, \quad f_{F_{i} P}^{X}: X_{P} \rightarrow X_{F_{i}}
$$

with the required composition properties. Clauses (i) and (ii) are immediate consequences of the definitions.

ProOF OF THEOREM 4.1. Let $\mathscr{P}_{n}=\{\sigma \in \mathscr{P} \mid \operatorname{dim} \sigma \leqslant n\}$ be the $n$-skeleton of $\mathscr{P}$. Define a morphism of the required type for the zero-skeleton by choosing a resolution of singularities $\pi_{v}: X_{v} \rightarrow Y_{v}$ for each vertex of $\mathscr{P}$. By induction assume that a surjection $\pi\left|\mathscr{P}_{n}: X\right| \mathscr{P}_{n} \rightarrow Y \mid \mathscr{P}_{n}$ exists which satisfies the theorem. To define $\pi$ and $X$ over $\mathscr{P}_{n+1}$, let $P$ be an $(n+1)$-polyhedron, and use the canonical extension diagram of the lemma to construct a preliminary version of $\pi$ and $X$. Let $X_{P}^{\prime}$ be a subvariety of $X_{P}$ of the correct dimension such that $\pi\left(X_{P}^{\prime}\right)=Y_{P}$, and let $X_{P}^{\prime \prime}$ be a resolution of singularities for $X_{p}^{\prime}$ with $\pi_{P}: X_{P}^{\prime \prime} \rightarrow Y_{P}$ the obvious map. Replace $X_{P}$ and $\pi_{P}$ by $X_{P}^{\prime \prime}$ and $\pi_{P}^{\prime \prime}$ to achieve the required extensions to the $(n+1)$-skeleton.

PROOF OF THEOREM 4.3. We begin as before with a resolution of singularities of the zero-skeleton and a preliminary extension from the $n$-skeleton to the $(n+1)$ skeleton. The definitive extension is constructed as follows: Let $Y_{F_{i}}^{*}$ be the subset of 
$Y_{F_{i}}$ over which $\pi_{F_{1}}$ is an isomorphism, and set

$$
Y_{P}^{\prime}=\left(f_{F_{i} P}^{Y}\right)^{-1}\left(Y_{F_{i}}^{*}\right), \quad X_{P}^{\prime}=\pi_{P}^{-1}\left(Y_{P}^{\prime}\right)
$$

Because $\pi_{F_{1}}$ is birational by construction, $Y_{F_{i}}^{*}$ is dense and open in $Y_{F_{i}}$. Because of the surjectivity of all maps in the above definitions, the property of being dense and open propagates to the inverse images, then to the intersection $Y_{P}^{\prime}$, and finally to $X_{P}^{\prime}$. Thus $X_{P}^{\prime}$ is a dense open on which $\pi_{P}$ is bijective. Let $X_{P}^{\prime \prime}$ be a smooth compactification to which $\pi_{P}$ extends as a morphism $\pi_{P}^{\prime \prime}$. Replace $X_{P}$ and $\pi_{P}$ by $X_{P}^{\prime \prime}$ and $\pi_{P}^{\prime \prime}$ to achieve the required extensions to the $(n+1)$-skeleton.

5. Mapping cyclinders. We define mapping cylinders for polyhedral complexes and spaces, then study birational morphism using the latter. For complexes the cylinder will be an object $\mathscr{C}=\mathscr{C}(l, r)$ associated to a diagram

$$
\mathscr{D}=[\mathscr{L} \stackrel{\prime}{\leftarrow} \stackrel{\sim}{\rightarrow} \mathscr{R}]
$$

of injective morphisms of complexes. When $\mathscr{D}$ looks like Figure 5.1, then the cylinder looks like Figure 5.2.

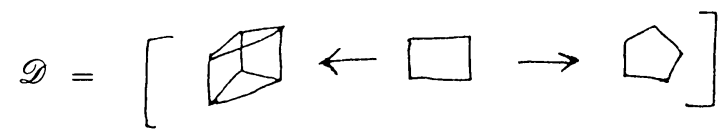

FIGURE 5.1

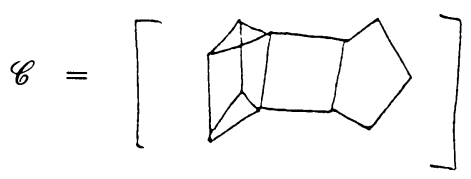

Figure 5.2

To give a formal definition, let $\mathscr{C}$ be a category which has one object and morphism for each object and morphism of $\mathscr{D}$.

OBJECTS.

$$
\begin{array}{ll}
\sigma \times\{0\} & \text { if } \sigma \in \mathscr{L}, \\
\sigma \times\{0,1\} & \text { if } \sigma \in \mathscr{M}, \\
\sigma \times\{1\} & \text { if } \sigma \in \mathscr{R} .
\end{array}
$$

MORPHISMS.

$$
\begin{array}{ll}
f_{\sigma \tau} \times \operatorname{id}_{\{0\}} & \text { if } \sigma, \tau \in \mathscr{L}, \\
f_{\sigma \tau} \times \operatorname{id}_{\{0,1\}} & \text { if } \sigma, \tau \in \mathscr{M}, \\
f_{\sigma \tau} \times \operatorname{id}_{\{1\}} & \text { if } \sigma, \tau \in \mathscr{R},
\end{array}
$$

where $\sigma<\tau$.

$$
\begin{array}{ll}
\sigma \times\{0\} \rightarrow l^{-1}(\sigma) \times[0,1] & \text { if } \sigma \in l(\mathscr{M}), \\
\sigma \times\{1\} \rightarrow r^{-1}(\sigma) \times\{0,1\} & \text { if } \sigma \in r(\mathscr{M}) .
\end{array}
$$

To fulfil the definitions completely, one must exhibit $\mathscr{C}$ as the category of a collection of convex polyhedra in a vector space $V$. To this end, suppose given

$$
|L| \subset V_{\mathscr{L}}, \quad|R| \subset V_{\mathscr{R}},
$$


and geometric maps

$$
|l|:|\mathscr{M}| \rightarrow|\mathscr{L}|, \quad|r|:|\mathscr{M}| \rightarrow|\mathscr{R}|
$$

Define a map

$$
H:|\mathscr{M}| \times[0,1] \rightarrow V_{\mathscr{L}} \times \mathbf{R} \times V_{R}=V_{\mathscr{d e f}}
$$

by

$$
H(x, t)=(1-t)(|l|(x), 0,0)+t(0,1|r|(x)),
$$

and observe that the images of $\sigma \times[0,1]$ and $\sigma \times\{t\}$ are convex polyhedra in $V$. The required collection is then

$$
\mathscr{L} \times\{0\} \times\{0\} \cup H(\mathscr{M} \times[0,1]) \cup\{0\} \times\{1\} \times \mathscr{R} .
$$

For polyhedral spaces we assume a diagram

$$
D=[L \stackrel{l}{\leftarrow} M \stackrel{r}{\rightarrow} R]
$$

whose underlying diagram of complexes is as above. The cylinder $C=C(l, r)$ will be a polyhedral space over $\mathscr{C}=\mathscr{C}(l, r)$ with one object and morphism for each object and morphism of $D$, defined in much the same way as before.

OBJECTS.

$$
\begin{array}{ll}
C_{\sigma \times\{0\}}=L_{\sigma} & \text { if } \sigma \in \mathscr{L}, \\
C_{\sigma \times[0,1]}=M_{\sigma} & \text { if } \sigma \in \mathscr{M}, \\
C_{\sigma \times\{1\}}=R_{\sigma} & \text { if } \sigma \in \mathscr{R} .
\end{array}
$$

MORPHISMS.

$$
\begin{array}{ll}
f_{\sigma \times\{0\}, \tau \times\{0\}}=f_{\sigma \tau}, & \sigma, \tau \in \mathscr{L}, \\
f_{\sigma \times[0,1], \tau \times[0,1]}=f_{\sigma \tau}, & \sigma, \tau \in \mathscr{M}, \\
f_{\sigma \times\{1\}, \tau \times\{1\}}=f_{\sigma \tau}, & \sigma, \tau \in \mathscr{R},
\end{array}
$$

where $\sigma<\tau$.

$$
f_{\sigma \times\{0\}, \sigma \times[0,1]}=l_{\sigma}, \quad f_{\sigma \times\{1\}, \sigma \times[0,1]}=r_{\sigma},
$$

where $\sigma \in \mathscr{M}$.

Now suppose given an epimorphism $\pi: X|\mathscr{P} \rightarrow Y| \mathscr{P}$. The base and exceptional loci fit together to give a diagram

$$
D(\pi)=[X \stackrel{i}{\leftarrow} E \stackrel{p}{\rightarrow} B]
$$

and hence define a canonical mapping cylinder $C(\pi)=C(i, p)$ over $\mathscr{P} \times I$, where $I$ is the complex consisting of the unit interval and its faces. Taking note of the commutative diagram below,

$$
\begin{array}{cccc}
X & \stackrel{i}{\leftarrow} & E & \stackrel{p}{\rightarrow}
\end{array}
$$


we see that $C(\pi)$ is canonically augmented by $Y$. The next result shows why the cylinder of an epimorphism is useful:

THEOREM 5.1. Let $\pi: X|\mathscr{P} \rightarrow Y| \mathscr{P}$ be an epimorphism, and let $C(\pi) \mid \mathscr{P} \times I$ be its canonical cylinder. Then the augmentation

$$
\varepsilon: C(\pi) \rightarrow Y
$$

induces a homotopy equivalence of realizations.

Proof. We will show that $|\varepsilon|$ has contractible fibers. To this end, let $z$ be a point of $|Y|$, and let $C(Y, z)$ be its carrier - the smallest polyhedral subspace of $Y$ such that $z \in|C(Y, z)|$. One may construct the carrier from the set $\beta \alpha^{-1}(z)$, where $\alpha$ and $\beta$ are the canonical projections below:

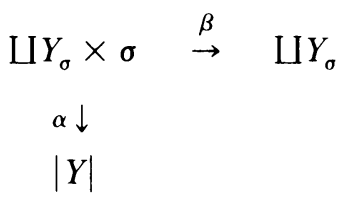

Let $C(X, z)=\pi^{-1} C(Y, z)$ and observe that the restriction of the canonical diagram to $C(Y, z)$ is as follows:

$$
C(X, z) \stackrel{i=}{i} \quad C(X, z) \stackrel{p}{\stackrel{p}{\rightarrow}} \quad C(Y, z)
$$

The fiber $|\varepsilon|^{-1}(z)$ is then the geometric cylinder for the restricted diagram. However, because $i$ is the identity and the realization of $C(Y, z)$ is a point, the cylinder $C\left(i_{z}, p_{z}\right)$ is a cone, hence contractible. Indeed, we have the following simple criterion for contractibility:

LEMMA 5.2. There is a canonical deformation retraction of the realization of

$$
C(F, \text { id })=[B \stackrel{F}{\leftarrow} A \stackrel{\text { id }}{\rightarrow} A]
$$

onto the realization of $B$.

Proof. Because the right-hand map is the identity, the geometric cylinder is the quotient of the space

$$
\tilde{C}=\coprod_{\sigma}\left(B_{\sigma} \times \sigma \amalg A_{\sigma} \times \sigma \times[0,1]\right)
$$

under the face relations. Define a deformation retraction of $\tilde{C}$ onto $\tilde{B}=\amalg B_{\sigma} \times \sigma$ by setting

$$
\begin{aligned}
\tilde{H}(x, \xi ; t) & =(x, \xi) \quad \text { for }(x, \xi) \in B_{\sigma} \times \sigma, \\
\tilde{H}(x, \xi, s ; t) & =(x, \xi, t s) \text { for }(x, \xi, s) \in A_{\sigma} \times \sigma \times[0,1] .
\end{aligned}
$$

One verifies that the deformation retraction $\tilde{H}: \tilde{C} \times[0,1] \rightarrow \tilde{C}$ of $\tilde{C}$ to $\tilde{B}$ is compatible with the face relations and so descends to a deformation retraction $H:|C| \times[0,1] \rightarrow|C|$ of $|C|$ to $|B|$. 
REMARK. All that we have done works equally well with the epimorphic (barred) variant of the base and exceptional locus. It is these that we shall use henceforth.

6. Polyhedral resolutions. We shall now prove a slightly refined version of Theorem 1.1 which guarantees the existence of smooth polyhedral resolutions. To this end, define the total dimension of a polyhedral space by

$$
\operatorname{tdim}(A \mid \mathscr{P})=\max \left\{\operatorname{dim} \sigma+\operatorname{dim} A_{\sigma} \mid \sigma \in \mathscr{P}\right\} .
$$

THEOREM 6.1. For every projective algebraic variety $X$ of dimension $n$ there is a smooth polyhedral resolution of total dimension $n$.

Proof. We begin with a technical definition. Let $I$ be the complex consisting of the unit interval and its faces, and let $I^{p}$ be the $p$-fold Cartesian product. In general the Cartesian product of polyhedral complexes makes sense: if $\sigma$ and $\tau$ are polyhedra in vector spaces $V$ and $W$, then $\sigma \times \tau$ is a polyhedron in $V \times W$. Given $X \mid I^{p}$, let $A X \mid I^{p}$ be the subspace defined by

$$
\begin{aligned}
& A X_{\{1\} \times \sigma}=X_{\{1\} \times \sigma}, \quad A X_{[0,1] \times \sigma}=X_{[0,1] \times \sigma}, \\
& A X_{\{0\} \times \sigma}=\operatorname{image}\left(X_{[0,1] \times \sigma} \rightarrow X_{\{0\} \times \sigma}\right) .
\end{aligned}
$$

In a suitably loose sense, $A X$ is the smallest subspace of $X$ which contains $X \mid(0,1] \times I^{p-1}$, where this latter means $X\left|I^{p}-X\right|\{0\} \times I^{p-1}$.

Our strategy is to construct a sequence of polyhedral resolutions $X \mid I^{p}$ of $X$ with the following properties:

(i) $p \geqslant 1$,

(ii) $X \mid\{0\} \times I^{p-1}$ is smooth,

(iii) $A X \mid I^{p}$ is epimorphic,

(iv) $\operatorname{gdim}\left(A X \mid I^{p}\right) \leqslant n-p$,

(v) there is a morphism $X\left|I^{p+1} \rightarrow X\right| I^{p}$ which induces a homotopy equivalence of realizations.

Since a polyhedral space of geometric dimension zero is smooth, $A X \mid I^{p}$ is smooth for $p$ sufficiently large but no greater than $n$. Since

$$
X \mid I^{p}=\left(X \mid\{0\} \times I^{p-1}\right) \bigsqcup\left(A X \mid(0,1] \times I^{p-1}\right)
$$

is a collection of spaces, $X \mid I^{p}$ is smooth for this value of $p$.

To implement the strategy, begin with a resolution of singularities, $\pi: \tilde{X} \rightarrow X$, and let

$$
X \mid I=C(\pi)=[\tilde{X} \leftarrow E \rightarrow B]
$$

where $A X \mid I=[E \leftarrow E \rightarrow B]$. The conditions required of $X \mid I$ are obviously satisfied.

To continue, suppose $X \mid I^{p}$ exists. Apply Theorem 4.2 to $A X \mid I^{p}$, taking note of the fact that it is epimorphic, to construct a birational morphism

$$
\pi_{A}: \widetilde{A X \mid} I^{p} \rightarrow A X \mid I^{p}
$$


with $\widetilde{A X}$ smooth and epimorphic. Use $\pi_{A}$ to define a resolution of singularities $\pi_{p}$ for $X \mid I^{p}$ by setting

$$
\begin{array}{ll}
\left(\tilde{X} \mid I^{p}\right)_{\sigma}=\left(X \mid I^{p}\right)_{\sigma} & \text { if } \sigma \in\{0\} \times I^{p-1} \\
\left(\tilde{X} \mid I^{p}\right)_{\sigma}=\left(\widetilde{A X} \mid I^{p}\right)_{\sigma} & \text { otherwise. }
\end{array}
$$

The face maps are defined in the natural way, with

$$
\begin{aligned}
& {\left[\left(\tilde{X} \mid I^{p}\right)_{[0,1] \times \tau} \rightarrow\left(\tilde{X} \mid I^{p}\right)_{\{0\} \times \tau}\right]} \\
& \quad=\left[\left(\widetilde{A X \mid} I_{[0,1] \times \tau}^{p} \rightarrow\left(\widetilde{A X \mid} I^{p}\right)_{\{0\} \times \tau} \rightarrow\left(X \mid I^{p}\right)_{\{0\} \times \tau}\right] .\right.
\end{aligned}
$$

The base and exceptional loci of $\pi_{A}$ are epimorphic and of geometric dimension at most $n-p-1$ by Proposition 4.2, and the same holds by construction for $\pi_{p}$. Thus, if we define $X \mid I^{p+1}=C\left(\pi_{p}\right)$, then conditions (i)-(v) are again satisfied, as required. Q.E.D.

7. Examples. We close with three examples of polyhedral resolutions which illustrate the theorem just proved.

A. A surface with an isolated singularity. Let $p$ be the singular point of $X$, and let $\tilde{X}$ be a resolution of singularities of $X$ with exceptional locus $E$. Then $X \mid I$ is given by the following labelled simplex:

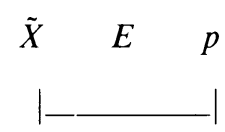

If $E$ is a smooth curve, $X \mid I$ is the required resolution; if not, one more step is necessary. Let $\Sigma$ be the singular locus of $E$, let $\tilde{E}$ be the normalization, and let $\tilde{\Sigma}$ be the lift of $\Sigma$ to $E$. From these, one assembles the resolution of $A X \mid I$ which is illustrated in Figure 7.1 below. The inclusions are for the center and exceptional locus.

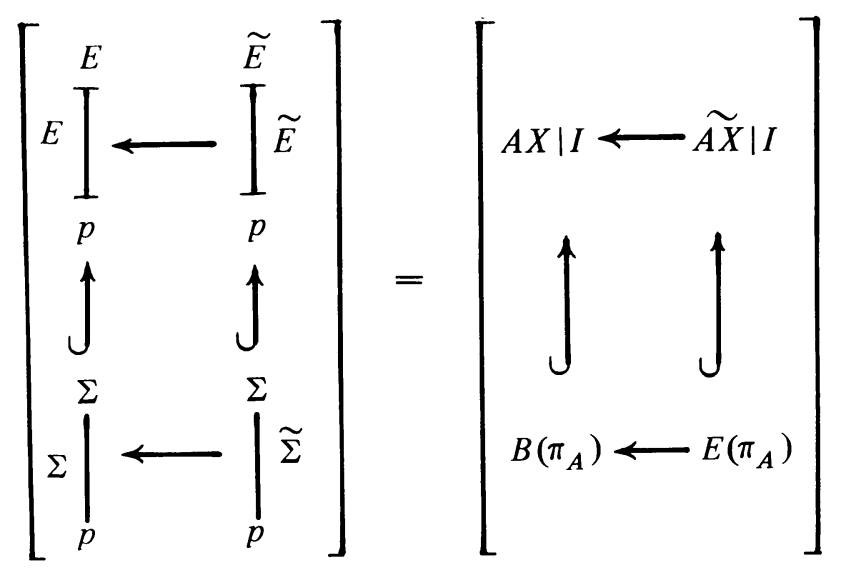

Figure 7.1 
The required resolution of $X$ is then given by the polyhedral space defined by Figure 7.2. With a different model for the polyhedral complex $I^{2}$, the polygon looks like Figure 7.3. Consolidation then produces the simplicial polyhedral space $X \mid T$ in Figure 7.4.

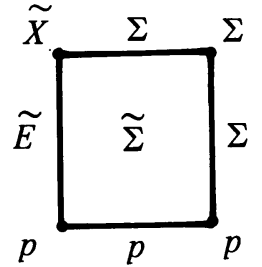

FIGURE 7.2

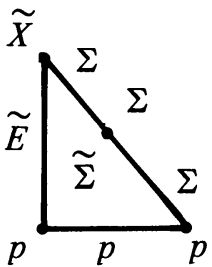

FIGURE 7.3

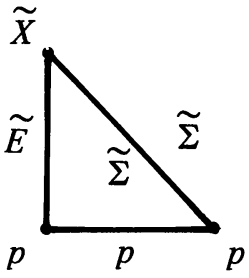

FIGURE 7.4

Since the realizations of $X \mid I^{2}$ and $X \mid T$ are homeomorphic, $X \mid T$ is also a resolution. As a simplicial space, it looks like Figure 7.5.

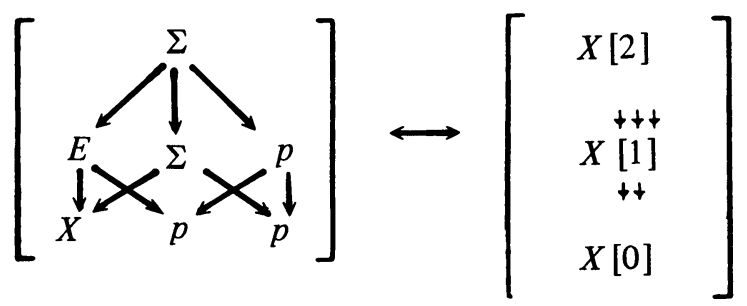

FIGURE 7.5

B. An arbitrary surface. An analogous procedure leads one to the simplicial polyhedral resolution in Figure 7.6. The $\Sigma$ 's have dimension one and the $T$ 's have dimension zero, where

(i) $\Sigma^{\prime}$ is the normalization of the singular locus $\Sigma$ of $X$,

(ii) if $\tilde{\Sigma}$ is the lift of $\Sigma$ to $X$, then $\tilde{\Sigma}^{\prime}$ is the normalization of $\tilde{\Sigma} \times_{\Sigma} \Sigma$,

(iii) $T$ and $T^{\prime}$ are the base and exception loci of $\Sigma^{\prime} \rightarrow \Sigma$,

(iv) $\tilde{T}$ and $\tilde{T}^{\prime}$ are the base and exceptional loci of $\tilde{\Sigma}^{\prime} \rightarrow \tilde{\Sigma}$.

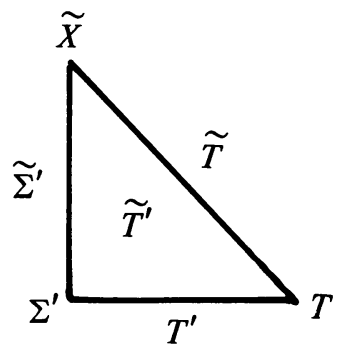

FigURE 7.6 
C. A curve in a smooth surface. The cohomology of the pair $(X, A)$ is that of the space $X / A$ obtained by collapsing $A$ in $X$ to a point. Because this space is the reduction of the mapping cone $C \mid I$,

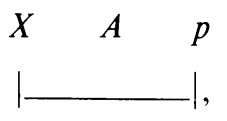

the cohomology of the cone as a polyhedral space is canonically isomorphic, via the map $C \mid I \rightarrow R(C \mid I)$ to the cohomology of the pair. If $A$ is smooth, then $C \mid I$ is also, and we are done. If not, let $\tilde{A} \rightarrow A$ be the normalization, with center and exceptional locus $\Sigma$ and $\tilde{\Sigma}$. Then the standard procedure leads to the smooth resolutions pictured in Figure 7.7.

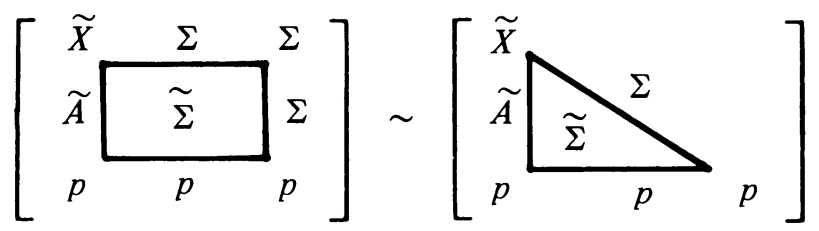

FigURE 7.7

\section{BIBLIOGRAPHY}

[C] James A. Carlson, Extensions of mixed Hodge structures, Jounées de Géométrie Algébrique d'Angers, Juillet 1979/Algebraic Geometry, Angers, 1979 (Arnaud Beauville, ed.), Sijthoff \& Noordhoff, Alphen den Rijn, The Netherlands, 1980, pp. 107-127.

[D] Pierre Deligne. Théorie de Hodge. III, Inst. Hautes Etude Sci. Publ. Math. No. 44, 1974, pp. 5-77.

Department of Mathematics, University of Utah, Salt Lake City, Utah 84112 